\title{
ARTICLE
}

Genetics and Genomics

\section{Molecular characterisation of aromatase inhibitor-resistant advanced breast cancer: the phenotypic effect of ESRI} mutations

\author{
Elena Lopez-Knowles ${ }^{1,2}$, Alex Pearson ${ }^{1}$, Gene Schuster ${ }^{1,2}$, Pascal Gellert ${ }^{1,2}$, Ricardo Ribas ${ }^{1}$, Belinda Yeo ${ }^{2,3,4}$, Ros Cutts ${ }^{1}$, Richard Buus ${ }^{1,2}$, \\ Isaac Garcia-Murillas ${ }^{1}$, Ben Haynes ${ }^{2}$, Lesley-Ann Martin ${ }^{1}$, Ian Smith ${ }^{3}$, Nick Turner ${ }^{1,3}$ and Mitch Dowsett ${ }^{1,2}$
}

BACKGROUND: Several thousand breast cancer patients develop resistance to aromatase inhibitors (Als) each year in the UK. Rational treatment requires an improved molecular characterisation of resistant disease.

MATERIALS AND METHODS: The mutational landscape of 198 regions in 16 key breast cancer genes and RNA expression of 209 genes covering key pathways was evaluated in paired biopsies before Al treatment and at progression on Al from 48 patients. Validity of findings was assessed in another five ESR1-mutated tumours progressing on Al.

RESULTS: Eighty-nine mutations were identified in 41 matched pairs (PIK3CA in 27\%; CDH1 in 20\%). ESR1 ( $n=5), E R B B 2(n=1)$ and MAP2K4 $(n=1)$ had mutations in the secondary sample only. There was very high heterogeneity in gene expression between Al-resistant tumours with few patterns apparent. However, in the ESR1-mutated Al-resistant tumours, expression of four classical oestrogen-regulated genes (ERGs) was sevenfold higher than in ESR1 wild-type tumours, a finding confirmed in the second set of ESR1-mutated tumours. In ESR1 wild-type Al-resistant tumours ERG expression remained suppressed and was uncoupled from the recovery seen in proliferation.

CONCLUSIONS: Major genotypic and phenotypic heterogeneity exists between Al-resistant disease. ESR1 mutations appear to drive oestrogen-regulated processes in resistant tumours.

British Journal of Cancer (2019) 120:247-255; https://doi.org/10.1038/s41416-018-0345-x

\section{INTRODUCTION}

Aromatase inhibitors (Als) are the standard of care as first-line treatment for postmenopausal women with oestrogen receptor positive $(E R+)$ advanced breast cancer (BC). ${ }^{1}$ However, the objective response rate to Als in the metastatic setting is between $20 \%-40 \%$ and virtually all patients eventually relapse with Alresistant disease. ${ }^{2,3}$ It is critical to understand the molecular drivers of the resistance to allow rational use of subsequent or concurrent therapy. Several potential mechanisms of resistance have been described including changes in the expression of ER or its coregulators, as well as the ESR1 mutational status. ESR1 mutations in the ligand-binding domain of ER lead to constitutive activity in model systems ${ }^{4}$ and have been detected in $15-20 \%$ of patients with metastatic $\mathrm{ER}+$ endocrine resistance $\mathrm{BC}^{5-10}$; up to $40 \%$ of patients have been reported to have ESR1-mutated circulating tumour (ct) DNA. ${ }^{11}$ Other potential mechanisms of resistance to endocrine therapy include the activation of signalling pathways such as the PI3K/mTOR pathway. ${ }^{12}$

Paired tumour biopsies before and at recurrence or progression on Als are infrequently available. However, in our previous report of 55 such pairs we found a highly variable immunohistochemical phenotype of several candidate markers between pre-Al and $\mathrm{Al}-$ resistant biopsies. ${ }^{13}$ Others ${ }^{14}$ have reported similar observations that indicate that multiple mechanisms of resistance occur to Al. While loss of ER occurred in some cases, others recurrences showed enhanced expression of ER suggesting persistent ER functioning but downstream markers of such functioning were not measured to confirm or refute this. Other biopsy pairs showed loss of PTEN or HER2 gain, which are consistent with experimental studies of resistance to oestrogen deprivation. ${ }^{15,16}$

To further investigate the range of molecular changes that are associated with Al-resistance, we analysed the same sample set ${ }^{13}$ using a targeted NGS panel to identify somatic mutation in 16 key genes and a Nanostring panel of 209 genes to identify changes in gene expression in major signalling pathways. We found that the majority of mutations in the Al-resistant tumour were shared with their paired pre-Al sample, but almost half of the pairs showed at least one private mutation. ESR1, ERBB2 and MAP2K4 had mutations in the secondary sample only, while there was no systematic difference between the primary and secondary sample for the other analysed genes. The expression of classically oestrogen-dependent genes that are downregulated in almost all Al-treated tumours ${ }^{17}$ supported a significant phenotypic impact of ESR1 mutations providing further evidence for the likely benefit from some therapeutic interventions.

\footnotetext{
${ }^{1}$ The Breast Cancer Now Toby Robins Research Centre at the Institute of Cancer Research, London, UK; ${ }^{2}$ Ralph Lauren Centre for Breast Cancer Research, Royal Marsden Hospital, London, UK; ${ }^{3}$ Breast Unit, Royal Marsden Hospital, London, UK and ${ }^{4}$ Olivia Newton-John Cancer Research Institute, Melbourne, VIC, Australia Correspondence: Mitch Dowsett (mitch.dowsett@icr.ac.uk)
} 


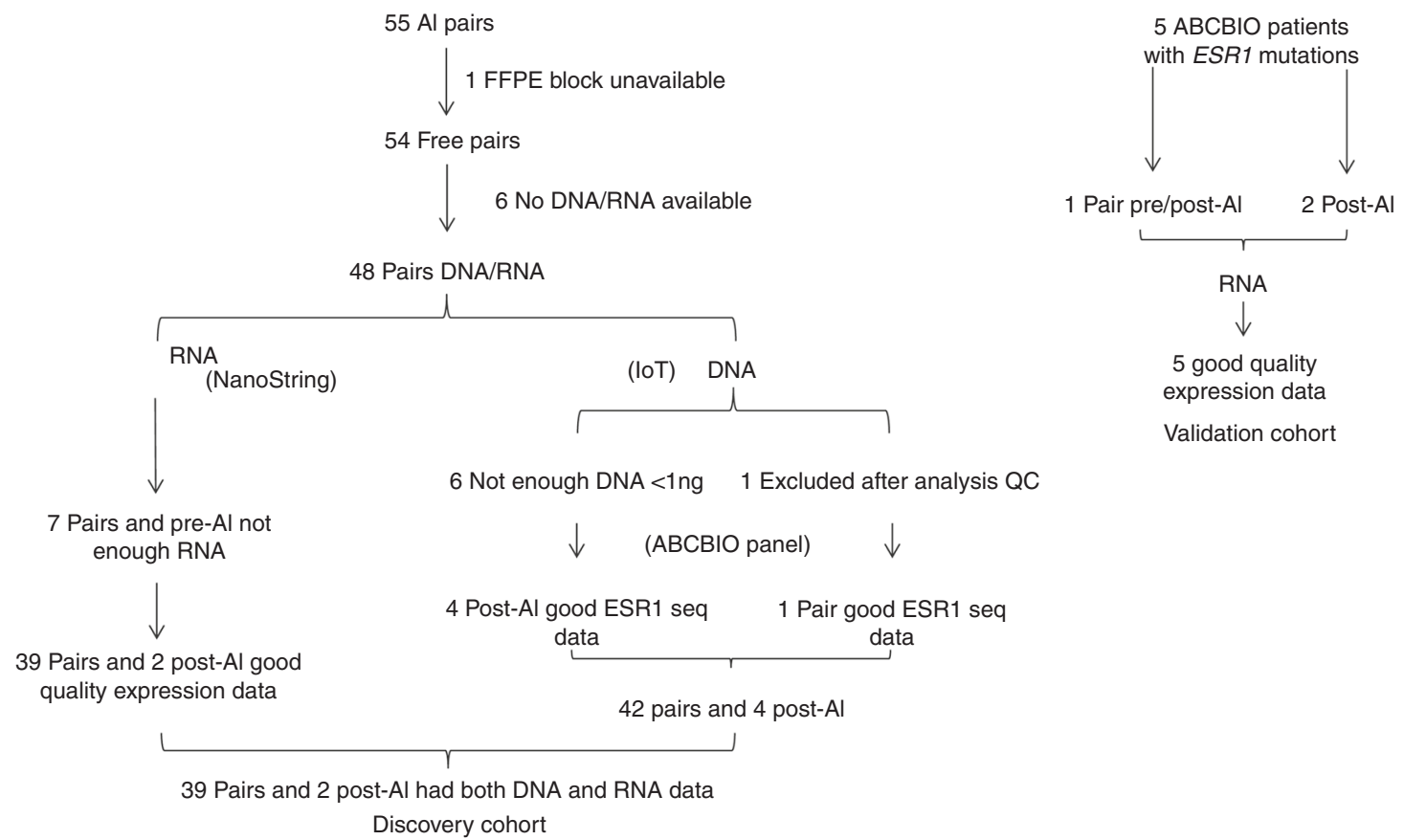

Fig. 1 Consort diagram of the $55 \mathrm{Al}$ paired samples (discovery cohort, left) and five ESR1 mutant samples from the ABC-BIO study (validation cohort, right)

\section{Materials and methods}

Patient selection and characteristics. Samples used in this study have been described previously. ${ }^{13}$ In brief, 55 patients with ER+ breast cancer from The Royal Marsden Hospital were retrospectively selected if they had relapsed or progressed during Al treatment in the locally advanced or metastatic setting (Discovery cohort, Fig. 1). Patient characteristics and clinical management are summarised in Table 1.37/48 (77\%) of patients received endocrine therapy prior to treatment with an $\mathrm{Al}$, with $31 / 48(65 \%)$ receiving tamoxifen. 5/48 (10\%) patients received both tamoxifen and an Al. Paired tissue blocks, pre and post-Al treatment, from 48 patients were available for DNA and RNA extraction. Of these 48 patients, a total of 21 patients received tamoxifen prior to the pre-Al sample being collected.

To assess the validity of observations made in the discovery cohort on the phenotype of tumours with ESR1 mutations, a set of biopsies from 5 patients with recurrent disease already known to have ESR1 mutations post-Al treatment was obtained from the $A B C-B I O$ study (Validation cohort, Fig. 1). The ABC-BIO study recruits patients at the Royal Marsden Hospital with advanced breast cancer with accessible metastatic deposits for DNA sequencing using the Breast NGS v1.1 probe set including probes to capture ESR1. Biopsies from three other patients in the ABC-BIO study that were known to harbour ESR1 mutations but had ceased Al treatment for at least 4 weeks prior to biopsy were excluded because of the potential impact on gene expression.

Essential details of molecular analysis are stated below and fully detailed in the supplementary materials.

DNA and RNA extraction. Patients had an FFPE tumour biopsy pre- and post-Al treatment. Tissue sections were microdissected and DNA and RNA were co-extracted using the AllPrep DNA/RNA FFPE Kit (Qiagen, Hilden, Germany), with an extended overnight digestion for the DNA extraction being the only modification from the manufacturer's instructions. Quantification was done using high sensitivity RNA and DNA Qubit assays (Thermo Fisher Scientific, Carlsbad, CA) and on a Bio-Rad QX200 droplet digital PCR (ddPCR) using RNAseP (Thermo Fisher Scientific). ${ }^{11}$ Samples from the validation cohort were also extracted following the same protocol; however, only one of five cases had a pre-Al treatment block available.

lon Torrent (IOT) PGM sequencing. DNA from the discovery cohort was amplified using a custom panel targeting 198 regions within 16 genes. These genes represent the most mutated genes in breast cancer. Five genes (CDH1, GATA3, MAP2K4, MAP3K1, PTEN) were covered between 73 and $100 \%$, while for the other 11 genes (AKT1, BRAF, ERBB2, ESR1, KIT, KRAS, PIK3CA, PIK3R1, RUNX1, SF3B1, TP53), amplicons for known hotspot regions were designed, resulting in a $100 \%$ coverage, except for ERBB2 (90\%) and RUNX1 (5\%). Libraries were prepared with $10 \mathrm{ng}$ of DNA and sequenced to a median depth of $782 \mathrm{X}$ using the lon Ampliseq Library Kit v2.0 (Thermo Fisher Scientific).

MiSeq and NextSeq sequencing. DNA from 5 tumours from the discovery cohort that were unsuccessful with lon Torrent and 8 from the validation cohort were run on the Miseq or NextSeq (Illumina, San Diego, CA) using the Breast NGS v1.1 probe set. Protocol and analysis details are described in supplementary materials. For the purposes of this report only ESR1 mutational data was extracted.

Mutational validation. Selected ESR1, TP53, HER2, MAP2K4, MAP3K1 and PIK3CA mutations were validated by droplet digital PCR (ddPCR) on a QX200 ddPCR system (Bio-Rad, Hercules, CA), with primers $(900 \mathrm{nM})$ and probes $(250 \mathrm{nM})$ and annealing temperatures described in Table S1. Cycling conditions and calculation of mutant concentration were described previously. $^{11,18}$

PIK3CA C420R and E418K and GATA3 K358fs mutations were validated by cycle sequencing.

Nanostring gene expression analysis. RNA was run on a NanoString nCounter ${ }^{\mathrm{TM}}$ with two custom gene expression panels that comprised of 194 genes in CodeSet 1 and 70 genes in CodeSet 2, according to manufacturer's guidelines. These were comprised of reference genes, the PAM50 gene set and genes involved in steroid hormone synthesis, ER targets, receptor tyrosine kinases, 


\begin{tabular}{|c|c|c|}
\hline Clinical characteristics & & $n(\%)$ \\
\hline \multicolumn{3}{|l|}{ Diagnosis } \\
\hline \multirow[t]{2}{*}{ Age (years) } & Mean & 54 \\
\hline & Range & $27-86$ \\
\hline \multirow[t]{3}{*}{ Disease status } & $\mathrm{EBC}$ & $41(85)$ \\
\hline & Locally advanced & $5(10)$ \\
\hline & Metastatic & $2(5)$ \\
\hline \multirow{2}{*}{$\begin{array}{l}\text { Age at start of Al treatment } \\
\text { (years) }\end{array}$} & Mean & 62 \\
\hline & Range & $33-88$ \\
\hline \multicolumn{3}{|l|}{ Pre-Al biopsy } \\
\hline \multirow[t]{3}{*}{ Site } & Primary & $30(62)$ \\
\hline & Local recurrence & $17(35)$ \\
\hline & Distant recurrence & $1(2)$ \\
\hline \multirow[t]{3}{*}{ Disease status } & $\mathrm{EBC}$ & $24(50)$ \\
\hline & Locoregional recurrence & $20(42)$ \\
\hline & MBC & $4(8)$ \\
\hline \multicolumn{3}{|l|}{ Al therapy b/w 1st and 2nd biopsy } \\
\hline \multirow[t]{3}{*}{ Type } & Letrozole & $25(52)$ \\
\hline & Anastrozole & $21(44)$ \\
\hline & Exemestane & $2(5)$ \\
\hline \multirow[t]{3}{*}{ Disease setting for Al therapy } & Adj/neoadj & $9(19)$ \\
\hline & Local recurrence & $25(52)$ \\
\hline & Metastatic & $14(30)$ \\
\hline \multicolumn{3}{|l|}{ Post-Al biopsy } \\
\hline \multirow[t]{3}{*}{ Site } & Primary & $7(15)$ \\
\hline & Local recurrence & $26(54)$ \\
\hline & Distant recurrence & $15(31)$ \\
\hline \multirow[t]{3}{*}{ Disease status } & $\mathrm{EBC}$ & $3(6)$ \\
\hline & Locoregional recurrence & $17(36)$ \\
\hline & MBC & $28(58)$ \\
\hline \multirow{4}{*}{$\begin{array}{l}\text { Endocrine therapy prior Al } \\
\text { treatment }\end{array}$} & None & $11(23)$ \\
\hline & Tamoxifen & $31(65)$ \\
\hline & Tamoxifen + Al & $5(10)$ \\
\hline & Grosrelin & $1(2)$ \\
\hline \multirow[t]{3}{*}{ Endocrine therapy after PD on Al } & Al & $31(65)$ \\
\hline & Tamoxifen & $7(15)$ \\
\hline & Fulvestrant & $5(10)$ \\
\hline \multirow[t]{2}{*}{ HER2 status of either tissues } & HER2 positive ${ }^{a}$ & $7(15)$ \\
\hline & Trastuzumab received & $6(13)$ \\
\hline \multirow[t]{2}{*}{ Overall survival ${ }^{\mathrm{b}}$ (years) } & Median & 8.75 \\
\hline & Range & $2-33$ \\
\hline \multicolumn{3}{|c|}{$\begin{array}{l}E B C \text { early breast cancer, } M B C \text { metastatic breast cancer, } A l \text { aromatase } \\
\text { inhibitor, } P D \text { progressive disease } \\
{ }^{2} \text { Either } 1 \text { st or } 2 \text { nd tissue sample } \\
\text { bDefined as time from first breast cancer diagnosis to death (alive patients } \\
\text { censored) }\end{array}$} \\
\hline
\end{tabular}

cell cycle/proliferation, apoptosis, cell signalling, mTOR and APOBEC (Table S2A and S2B). Intrinsic subtypes were identified by NanoString Technologies using a proprietary algorithm. NanoString was performed for 39 pairs and 2 post-Al samples from the discovery cohort and 1 pair and 2 post-Al from the validation cohort.
Statistical analysis. Statistical tests were performed as indicated using either R v3.2.3 or Graphpad Prism v7. $P$-value $<0.05$ was considered statistically significant. Where appropriate paired analyses were performed.

\section{RESULTS}

Discovery cohort

Population. A consort diagram showing the sample availability in the population is provided in Fig. 1. The clinicopathological characteristics of the 48 sample pairs with adequate either DNA and/or RNA data are shown in Table 1. In summary, the first tissue sample (pre-Al) was taken most frequently $(62 \%)$ from the primary BC or from a local recurrence (35\%). At the time of this sample, $50 \%$ of patients had early disease, $42 \%$ had loco regional relapsed disease and $8 \%$ had metastatic BC. The second, post-Al tissue was most frequently (54\%) from a site of local recurrence. At the time of the post-Al tissue, $58 \%$ of patients had metastatic disease, $36 \%$ had loco regional recurrence and for $6 \%$ of patients the post-Al tissue represented progression in the primary after neoadjuvant Al.

Ion Torrent mutational landscape. Using stringent criteria (see supplementary material), we identified a total of 89 somatic mutations (47 unique genomic positions) among the 41 pairs of sample with adequate DNA and that passed QC, Table S3). The mutations are shown for individual patients in Fig. 2 along with data on PAM50 subtype and previously reported IHC status for ER, PgR, PTEN, Ki67 and HER2 (FISH as necessary). Across all samples, 36 mutations were found in both the primary and secondary samples (shared mutations) whilst 18 mutations were private to one sample of the pair (Fig. S1). For the mutations that were identified in both paired samples, there was no significant difference in variant allele frequency (VAF) between the samples (data not shown). For many pairs we found at least one mutation with high VAF in both samples suggesting a common founding clone. There was no significant difference between the total number of mutations identified on the pre and post samples. The most frequently mutated gene was PIK3CA (27\%) followed by CDH1 (20\%). Three genes: ERBB2 (L755S), MAP2K4 (located at Intron 9-10) and ESR1 (D538G and E380Q) were mutated exclusively in the post sample and were exclusive of each other. Mutations were validated by ddPCR and cycle sequencing (Table S4) with identified VAFs similar to those found by sequencing, demonstrating high reproducibility of the data. Of the 12 sample pairs with no mutations detected, three were HER2 positive and four had a marked decrease of ER staining in the post-Al sample. Both of these phenotypes might lead to less selective pressure for the acquisition of mutations.

ESR1 mutations. To complement the ESR1 mutational analysis five further samples from the discovery cohort that were unsuccessful with lon Torrent were run with an NGS Breast v1.1 panel (Supplementary Materials). This identified one additional ESR1 mutation in a post-Al sample. This mutation was a previously unreported substitution followed by an insertion at the aa536 hotspot of known mutations (L536indelGV). In all of the five patients with ESR1 mutations the resistant biopsy was in the metastatic setting (Fig. S2). In one of these cases (patient 23) an intermediate sample taken after 5 years of tamoxifen in the metastatic setting and before Al treatment was available and was found to be ESR1 wild type.

Gene expression. For five genes both $\mathrm{IHC}$ and gene expression data (Table S5) were available and for all of these there was a strong significant correlation between the two measurements (Table S6). ${ }^{19}$

Two-way hierarchical clustering of the global gene expression in the pre- and post-Al groups showed 38\% (15/39) of pairs clustered 


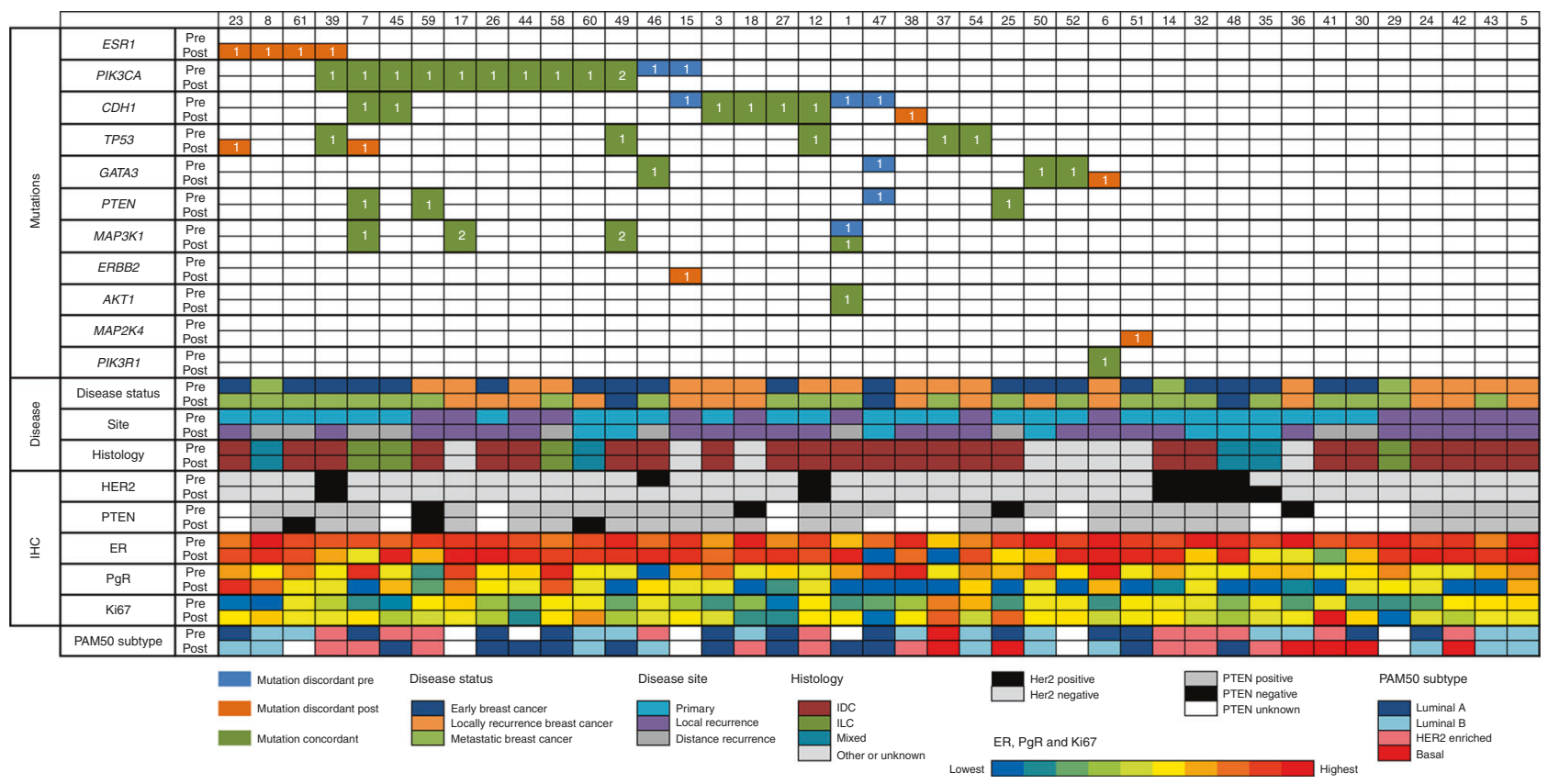

Fig. 2 Mutation matrix. All somatic mutations in the coding sequence (CDS) are shown together with IHC expression, clinicopathological parameters and PAM50 subtypes. 1 and 2 indicate the number of mutations identified

together (Fig. 3a). Thirty-six pairs (plus two pre- and two post-Al samples) had PAM50 subtype calculated (Table S7). Only $56 \%$ of sample pairs maintained their PAM50 subtype at progression after Al treatment (Table S8). Of particular note only one case was classified as basal-like at baseline but six were classified as basallike at resistance. Low expression of oestrogen response genes were a consistent feature of this group. The clustering shows some distinct patterns with three major branches labelled $A, B$ and $C$ in Fig. 3a. Branch A consists largely of luminal $A$ and luminal $B$ samples with substantial heterogeneity between them. Branch B consists mainly of HER2-enriched samples and some luminal B. In contrast branch $C$ contains all of the basal-like samples, most of which were unpaired post-treatment samples. The proliferation group of genes appeared to be the dominant feature in clustering the samples most notably into 2 sub-clusters of branch $C$.

Figure $3 \mathrm{~b}$ shows 2-way hierarchical clustering of just the Alresistant samples. While four main clusters can be recognised, the very wide heterogeneity in gene expression in these samples is evident with few groupings due to consistent patterns of expression across the gene set. A small group of tumours with basal-like features (branch A) again segregated from the others based mainly on low expression of oestrogen-regulated genes and high expression of genes in the immune cluster. The central two clusters ( $B$ and $C$ ) in Fig. 3b differ from the others mainly by their higher expression of oestrogen-regulated genes and contain the ESR1-mutated tumours (see below). The segregation of clusters $B$ and $C$ from one another is then related mainly to proliferation-associated genes. Notably, those with the relatively high proliferation were associated with relatively high signal transduction and immune signalling. The segregation of the cluster classified as HER2-enriched was unexpectedly not dependent on high levels of genes associated with signal transduction but rather on either relatively high proliferation or relatively low expression of immune-related genes.

Eighteen genes were significantly (FDR 5\%) downregulated and one (TBP) was upregulated at progression after Al (Fig. 4). Ten of the 13 most markedly downregulated were known to be subject to regulation by oestrogen signalling. After exclusion of ER negative samples $13 / 18$ genes were significantly differentially expressed. The five genes no longer significantly different were TFF3, SCUBE2, SLC39A6, TBP, PIK3R2 and GATA3. This indicates that suppression of a major axis of oestrogen regulation is maintained despite these tumours demonstrating clinical resistance to Al. Further, expression of ESR1 and ERa show a strong correlation with the significantly differentially expressed genes (Fig. S3A). The discovery cohort is phenotypically heterogeneous, yet unsupervised clustering of the 18 differentially expressed genes reveals robust downregulation of ERGs in the majority of tumours (Fig. S3B).

Twenty-one patients with paired samples, of which 16 have expression data, had received tamoxifen prior to the pre-Al sample being collected and conceivably this could have impacted on the expression of these 18 differentially regulated genes in the pre-Al sample. However there was no significant difference in gene expression for any of the genes according to prior tamoxifen treatment (Fig. S4). This lack of effect of prior tamoxifen may be due to the drug's partial agonist activity which is marked in postmenopausal women. ${ }^{20}$

ESR1 mutation and gene expression. There was no significant difference in expression of four oestrogen-regulated genes (TFF1, GREB1, PDZK1 and PgR) that we have previously used as markers of oestrogenic signalling, ${ }^{17}$ in the pre-Al samples from the five patients in the discovery cohort that went on to acquire an ESR1 mutation compared with those that did not (Fig. S5). In four of the five cases it was notable however that oestrogen-regulated gene expression was in the upper range of that in all samples. Expression of the four oestrogen-regulated genes in post-Al samples with ESR1 mutations was on average more than twofold higher than in ESR1 wild-type samples for individual genes, and the average expression of these genes in post-Al samples with ESR1 mutations was more than 6-fold higher than in post-Al samples with wild-type ESR1 (MannWhitney $P=0.006$, Fig. S5).

We used the validation cohort to assess the consistency of these observations of a relationship between oestrogen-regulated gene expression and ESR1 mutations. This cohort consisted of an additional five metastatic samples with previously described ESR1 mutation in a sample taken after Al treatment increasing the number of ESR1-mutated cases with gene expression data to 10 . 
a

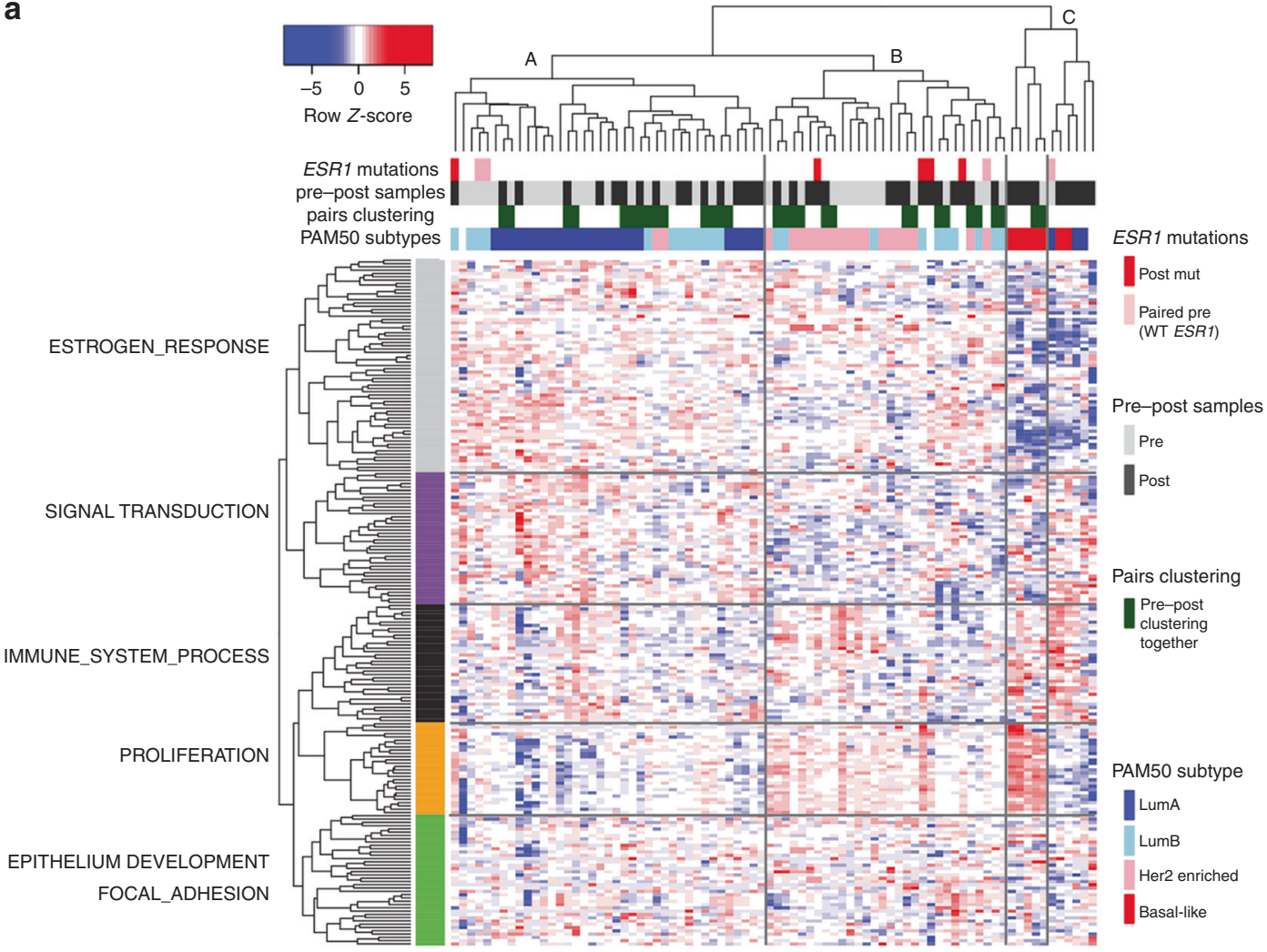

b

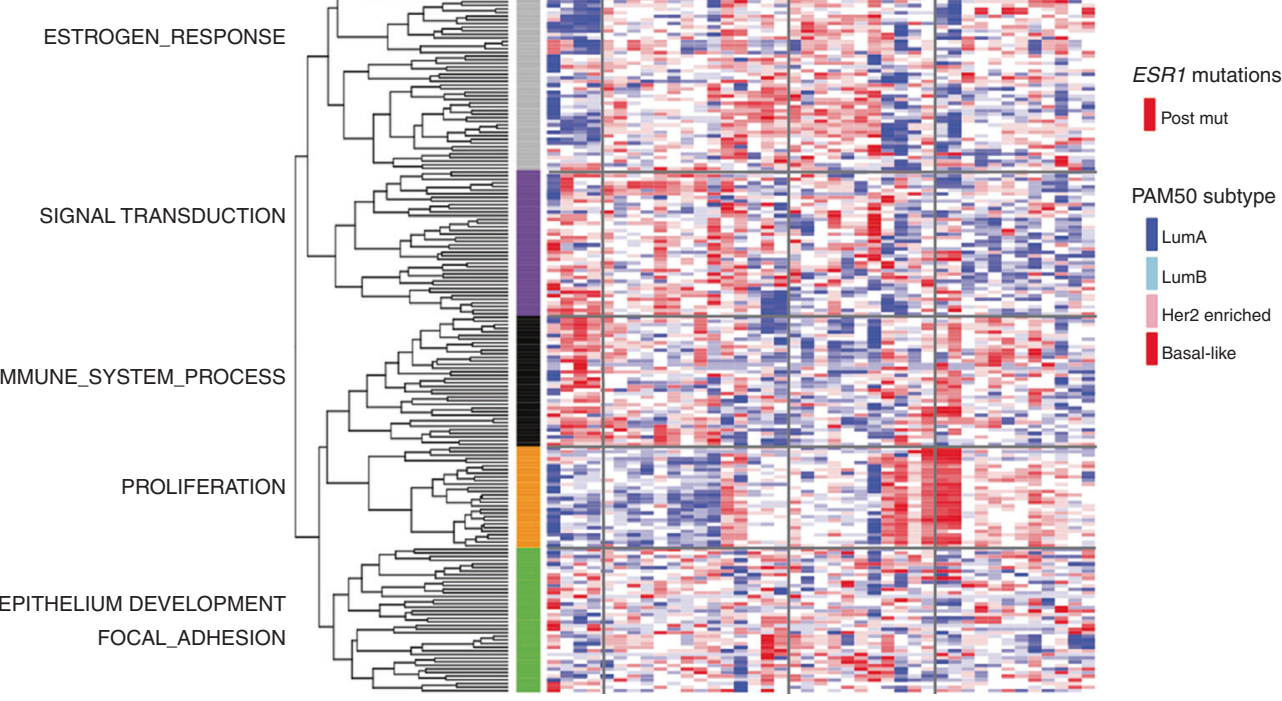

Fig. 3 a Hierarchical clustering of the 39 sample pairs and two unpaired post samples by gene expression. ESR1 mutational status, pair preand post-Al status (together with pair clustering) and PAM50 subtypes are indicated at the top of the cluster. Five gene (row) clusters are annotated by most significant terms generated from compute overlaps analysis in Broad Institute GSEA website (http://software. broadinstitute.org/gsea/msigdb/annotate.jsp). b Hierarchical clustering of the 41 post samples by gene expression. ESR1 mutational status and PAM50 subtypes are indicated at the top of the cluster. Five gene (row) clusters were taken from clustering used in Fig. 2 

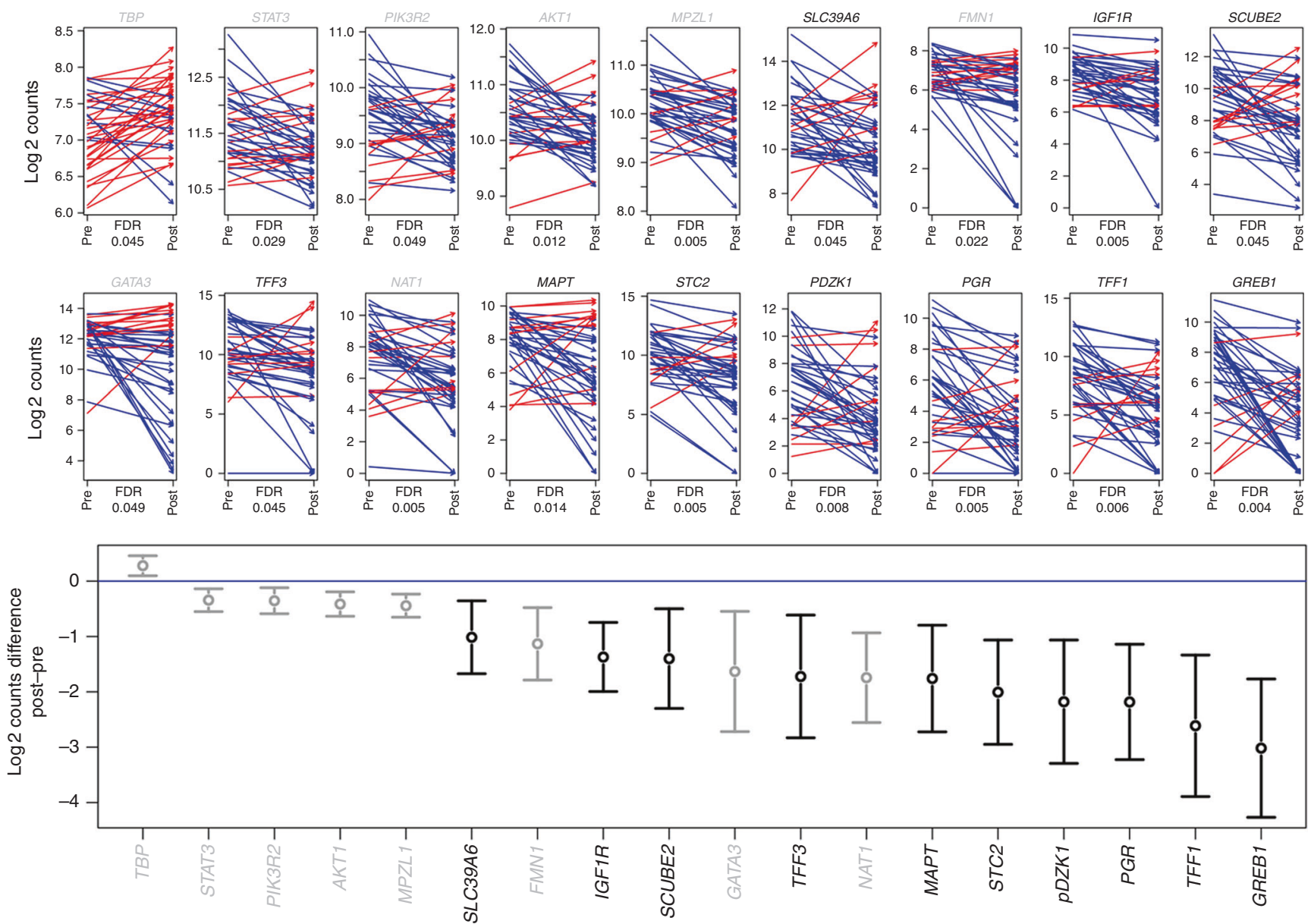

Fig. 4 Upper panel, arrow plot of 18 genes that changed significantly pre- and post-Al. Red arrows identify increase of expression in the paired post sample and blue arrows a decrease in expression. FDR values for Student's $t$-test are shown. Lower panel, box plots of the same 18 genes with mean and $95 \%$ confidence interval of log2 difference between paired pre and post samples. Genes coloured in black are ERG genes

The clinicopathological characteristics of the samples (1 pair and 4 Post-Al samples) are shown in Table S9 and the treatment chronology from diagnosis to death is shown in Fig. S6.

Gene expression of 33 genes was significantly different in the progression sample between ESR1 wild-type and the ten-mutated tumours (Fig. S7). FOXO3a was the only gene observed to have lower expression in ESR1 mutant post-Al samples. Using Fisher's exact test, the remaining 32 genes with higher expression in ESR1 mutant post-Al samples were significantly enriched for annotations associated with proliferation and most markedly with oestrogen regulation. Five of the genes are part of the 11-gene proliferation signature in PAM50 ${ }^{21}(P=0.02$, fisher exact test), and 11 are oestrogen-regulated (GSEA Molecular Signature Database Hallmark of Oestrogen Response Early/Late, ${ }^{22} P=0.01$, fisher exact test). In addition, two of these genes (MELK and BIRC5) are associated with worse outcome or metastasis. ${ }^{23,24}$ After exclusion of ER negative samples, 25/33 genes were significantly differentially expressed, including 8/10 ERGs and the five genes from the PAM50 proliferation signature. The eight genes no longer significantly different were IL6ST, PGR, FOXO3A, FKBP4, HRAS, KIF2C, CXXC5 and RPLPO.

Figure $5 \mathrm{a}$ shows the associations between oestrogen-regulated gene (ERG) expression and ESR1 mutational status between all ten ESR1-mutated cases and the non-mutated cases according to baseline or post-treatment status. Post-Al samples with ESR1 mutations had more than sevenfold higher ERG expression than post-Al wild-type samples (MannWhitney $P=1.7 \mathrm{e}-6$ ). Figure $5 b$ shows no significant differences in the PAM50 proliferation genes between the post-treatment samples according to ESR1 mutation status. A linear scale plot emphasises the magnitude of the difference in ERG expression between post-Al samples with or without ESR1 mutation (Fig. S8) and the separation in the samples according to ERG expression is particularly clear when shown in a waterfall plot (Fig. 5c). It is notable that the post-AI ESR1-mutated tumour with the lowest oestrogen-regulated expression carried an E380Q mutation and was also HER2-positive though this is the only ESR1-mutated sample with HER2 overexpression making the importance of its association with low ERG expression uncertain.

\section{DISCUSSION}

Several thousand women diagnosed with ER + breast cancer recur each year with endocrine resistant disease. The majority are postmenopausal and almost all will have received an Al before or after their recurrence and will require management of their Alresistant disease. Many potential mechanisms have been reported in model systems but few of these have been confirmed as being associated with Al-resistance in the clinic. To a large degree this is because tissues are difficult to acquire in which to study such associations. The collection of paired pre-Al and Al-resistant 

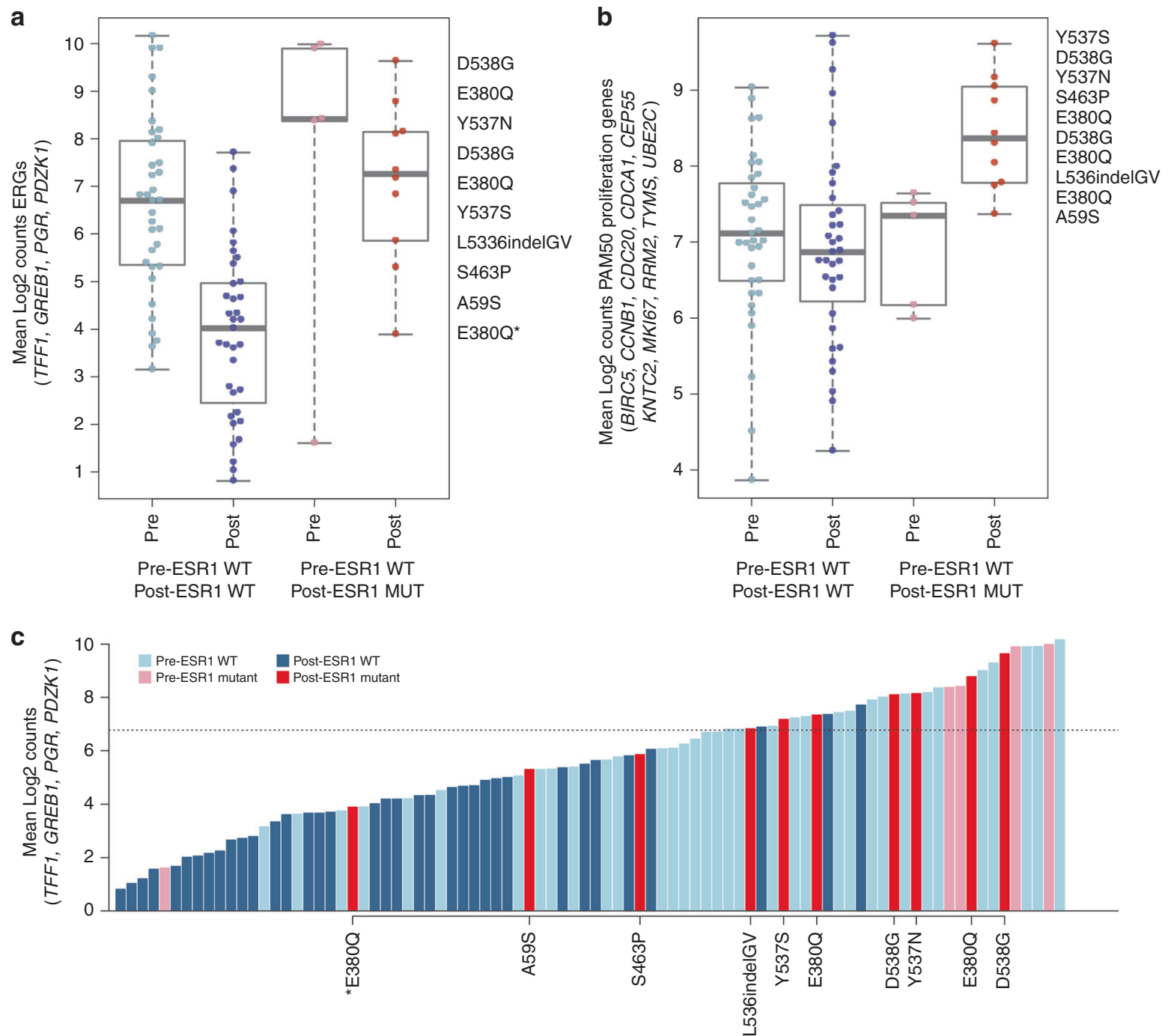

Fig. 5 a ESR1 mutations and avERG expression. Box plots of the average expression of TFF1, GREB1, PgR and PDZK1 are shown in the Pre- and Post-Al samples in ESR1 WT and 13 MUT samples (five from Al study and eight from additional cohort). b ESR1 mutations and PAM50 proliferation gene expression. Box plots of the average expression of the PAM50 proliferation genes are shown in the Pre- and Post-AI samples. c Waterfall plot of ESR1 mutational status and ERG expression. The dashed line represents the mean of all Pre samples. ${ }^{*}$ Indicates a Post-AI ESR1 mutant sample that is HER2 positive

tissues assessed here for mutational status and expression levels of $\mathrm{BC}$ associated genes although modest in size is therefore an uncommon cohort. Our earlier report revealed very marked heterogeneity between resistant tumours in key IHC biomarkers. ${ }^{12}$ Of note, ER expression was maintained or enhanced in the majority of tumours and was felt to be consistent with a potential for oestrogen signalling in the face of $\mathrm{Al}$ to be a driver of resistance, a mechanism that is supported in only a minority of $\mathrm{ER}+$ resistant tumours in the current study.

Our data support those from more wide-ranging studies of metastatic breast cancer, in that there was an absence of observed major increases in the acquisition of driver mutations in metastases $^{10,25,26}$ at least among the selected panel of frequently mutated genes assessed. The only gene that differed substantially was ESR1 in which mutations have been described to be markedly enriched in metastases after Al treatment. ${ }^{5,7-10}$ In this study we identified ESR1 mutations in $11 \%$ of patients, which is at lower end of the reported frequency. This may be due to many of our samples being local recurrences.
ESR1 mutated recurrent breast cancer has become a focus of attention in the possible development of new agents, such as selective oestrogen receptor degraders but very little has been reported on the phenotype of the ESR1-mutated tumours. Evidence from model systems indicates the ligand-independent activity of the hotspot ESR1 mutations. ${ }^{4,27-29}$ Our clinical data on the significantly higher expression of ERGs when ESR1 mutations were present, despite the on-going treatment with $\mathrm{Al}$, supports this being valid in clinical tissues. While our observation was made on a relatively small number of samples, it was validated by examination of another cohort from an on-going study of the clinical importance of mutations in metastatic breast cancer. The co-association of the high ERG expression and high proliferation genes in the ESR1-mutated tumours is consistent with the tumour progression being at least partly driven by the mutations. In contrast, the continued suppression of the ERG expression in tumours in which mutations were not detected implies a disconnect between proliferation and oestrogen signalling. Persistent suppression of ERG expression is clearly not a signal for continued anti-tumour effectiveness of the Al: 
assessment of these genes as a pharmacodynamics marker in this instance would likely be misleading.

We observed small numbers of other mutations that could underpin resistance in individual patients. These included a MAP2K4 mutation which likely disrupts splicing and potentially leads to not recognising exon 9 by the spliceosome or retaining the intron downstream of exon 9 and the ERBB2 L755S which has been previously associated with lapatinib resistance ${ }^{30}$ but has also been associated with response to the alternative HER2 tyrosine kinase inhibitor, neratinib. ${ }^{31}$

PIK3CA and TP53 are the most commonly mutated genes in BC with over $30 \%$ of patients carrying mutations in either of these genes (IntOgen database ${ }^{32}$ ). In our study we found that $27 \%$ of the patients had mutations in one or both of their samples in PIK3CA, but only $15 \%$ had a TP53 mutation (likely due to targeting of TP53 hotspots in our targeted panel). We also found many patients with a $\mathrm{CDH} 1$ mutation (20\%). Loss of $\mathrm{CDH} 1$ is a common feature of lobular breast cancer which is almost always $\mathrm{ER}+. \mathrm{CDH} 1$ controls the cellular adhesion dynamics ${ }^{33}$ and its loss has been associated with increased cancer invasion. ${ }^{34}$ These features might explain the unusually high frequency in this selection of patients, all of whom relapsed after Al treatment.

There was little consistency other than marked downregulation of ERGs in most patients in recurrent samples. PAM50 subtypes were maintained in $>55 \%$ of patients in agreement with the $61 \%$ recently described in matched primary and metastatic pairs. ${ }^{5}$ The meaning of the intrinsic subtypes in metastatic disease is however unclear particularly when, as in this study, transcriptional features that underpin the subtyping are impacted by medical therapy.

The most notable feature of the gene expression analyses was the very high degree of heterogeneity between recurrent tumours; this was apparent even within the three or four main clusters identified. This does not necessarily imply that gene expression profiling of recurrent tumours is without value. Rather it supports the need for individualised interpretation of profiles for individual tumours. This is especially so with regard to features such as oestrogen regulation, that might imply the likely benefit or not of alternative targeting of oestrogen signalling, or individual signal transduction pathways that align with particular inhibitors.

Some weaknesses in the current study need to be considered. Many patients had received chemotherapy or tamoxifen prior to the pre-Al sample and then progressed after being treated with an Al. Although prior treatment with tamoxifen might have been expected to impact on gene expression, particularly of known oestrogen-regulated genes, our analyses revealed no significant effect of this prior treatment on the main gene changes noted. Our mutational and transcriptional characterisation was based around features known to be of relevance in breast cancer. An assessment at a more genome-wide level would require a much larger sample set to have confidence in novel observations.

In summary, there is major inter-tumour heterogeneity of genotypic and phenotypic features that may drive resistance to Als in recurrent breast cancer, requiring highly individualised interpretation of likely dominant pathways in particular cases. Mutational analysis of recurrent disease is of value in identifying targetable abnormalities. Mutations in ESR1 gene are frequently acquired in recurrent disease, having enhanced ERG expression alongside high proliferation-associated genes provides a strong rationale for their targeting with novel agents targeted at the degradation of ligand-independent ER.

\section{ACKNOWLEDGEMENTS}

We would like to thank Monica Arnedos for putting together this sample group and Maria Afentakis and Margaret Hills for performing the IHC analysis. This work was supported by Breast Cancer Now, working in partnership with Walk the Walk; and the
National Institute of Health Research Royal Marsden/Institute of Cancer Research Biomedical Research Centre.

\section{AUTHORS CONTRIBUTIONS}

E.L.K. and A.P. performed experiments and wrote the manuscript, G.S. and P.G. analysed the data, R.R. performed loT experiment, B.Y. helped with study demographics, R.C. analysed loT data, R.B. and B.H. helped with NanoString, I.G.M. helped with ddPCR, L.A.M., I.S., N.T. helped write the manuscript, M.D. designed study and wrote the manuscript.

\section{ADDITIONAL INFORMATION}

Supplementary information is available for this paper at https://doi.org/10.1038/ s41416-018-0345-x.

Competing interests: The authors declare no competing interests.

Ethics approval and consent: The project was approved under the National Research Ethics Service (approval number: 08/H0801/111) and the Royal Marsden Committee for Clinical Research (number 3002).

\section{REFERENCES}

1. Cardoso, F. et al. Locally recurrent or metastatic breast cancer: ESMO Clinical Practice Guidelines for diagnosis, treatment and follow-up. Ann. Oncol. 21(Suppl 5), v15-v19 (2010).

2. Johnston, S. R. New strategies in estrogen receptor-positive breast cancer. Clin. Cancer Res. 16, 1979-1987 (2010).

3. Mehta, R. S. et al. Combination anastrozole and fulvestrant in metastatic breast cancer. N. Eng. J. Med. 367, 435-444 (2012).

4. Martin, L. A. et al. Discovery of naturally occurring ESR1 mutations in breast cancer cell lines modelling endocrine resistance. Nat. Commun. 8, 1865 (2017).

5. Fumagalli, D. et al. Somatic mutation, copy number and transcriptomic profiles of primary and matched metastatic estrogen receptor-positive breast cancers. Ann. Oncol. 27, 1860-1866 (2016).

6. Jeselsohn, R., Buchwalter, G., De Angelis, C., Brown, M. \& Schiff, R. ESR1 mutationsa mechanism for acquired endocrine resistance in breast cancer. Nat. Rev. Clin. Oncol. 12, 573-583 (2015).

7. Jeselsohn, R. et al. Emergence of constitutively active estrogen receptor-alpha mutations in pretreated advanced estrogen receptor-positive breast cancer. Clin. Cancer Res. 20, 1757-1767 (2014).

8. Robinson, D. R. et al. Activating ESR1 mutations in hormone-resistant metastatic breast cancer. Nat. Genet. 45, 1446-1451 (2013).

9. Toy, W. et al. ESR1 ligand-binding domain mutations in hormone-resistant breast cancer. Nat. Genet. 45, 1439-1445 (2013).

10. Yates, L. R. et al. Genomic evolution of breast cancer metastasis and relapse. Cancer Cell. 32, 169-184 e167 (2017).

11. Schiavon, $G$, et al. Analysis of ESR1 mutation in circulating tumor DNA demonstrates evolution during therapy for metastatic breast cancer. Sci. Transl. Med. 7, 313ra182; https://doi.org/10.1126/scitransImed.aac7551 (2015).

12. Miller, T. W., Balko, J. M. \& Arteaga, C. L. Phosphatidylinositol 3-kinase and antiestrogen resistance in breast cancer. J. Clin. Oncol. 29, 4452-4461 (2011).

13. Arnedos, M. et al. Biomarker changes associated with the development of resistance to aromatase inhibitors (Als) in estrogen receptor-positive breast cancer. Ann. Oncol. 25, 605-610 (2014).

14. Criscitiello, C. et al. Biopsy confirmation of metastatic sites in breast cancer patients: clinical impact and future perspectives. Breast Cancer Res. 16, 205 (2014).

15. $\mathrm{Fu}, \mathrm{X}$. et al. Overcoming endocrine resistance due to reduced PTEN levels in estrogen receptor-positive breast cancer by co-targeting mammalian target of rapamycin, protein kinase $\mathrm{B}$, or mitogen-activated protein kinase kinase. Breast Cancer Res. 16, 430 (2014).

16. Massarweh, $\mathrm{S}$. et al. Mechanisms of tumor regression and resistance to estrogen deprivation and fulvestrant in a model of estrogen receptor-positive, HER-2/neupositive breast cancer. Cancer Res. 66, 8266-8273 (2006).

17. Dunbier, A. K. et al. Molecular profiling of aromatase inhibitor-treated postmenopausal breast tumors identifies immune-related correlates of resistance. Clin. Cancer Res. 19, 2775-2786 (2013).

18. Lopez-Knowles, E. et al. Relationship of PIK3CA mutation and pathway activity with antiproliferative response to aromatase inhibition. Breast Cancer Res. 16, R68 (2014). 
19. Martin, M. et al. PAM50 proliferation score as a predictor of weekly paclitaxel benefit in breast cancer. Breast Cancer Res. Treat. 138, 457-466 (2013).

20. Dowsett, M. \& Howell, A. Breast cancer: aromatase inhibitors take on tamoxifen Nat. Med. 8, 1341-1344 (2002).

21. Nielsen, T. O. et al. A comparison of PAM50 intrinsic subtyping with immunohistochemistry and clinical prognostic factors in tamoxifen-treated estrogen receptor-positive breast cancer. Clin. Cancer Res. 16, 5222-5232 (2010).

22. Subramanian, A. et al. Gene set enrichment analysis: a knowledge-based approach for interpreting genome-wide expression profiles. Proc. Natl Acad. Sci. USA 102, 15545-15550 (2005).

23. Hamy, A. S. et al. BIRC5 (survivin): a pejorative prognostic marker in stage II/III breast cancer with no response to neoadjuvant chemotherapy. Breast Cancer Res. Treat. 159, 499-511 (2016).

24. Speers, C. et al. Maternal embryonic leucine zipper kinase (MELK) as a novel mediator and biomarker of radioresistance in human breast cancer. Clin. Cancer Res. 22, 5864-5875 (2016).

25. Siegel, M. B. et al. Integrated RNA and DNA sequencing reveals early drivers of metastatic breast cancer. J. Clin. Invest. 128, 1371-1383 (2018).

26. Gibson, W. J. et al. The genomic landscape and evolution of endometrial carcinoma progression and abdominopelvic metastasis. Nat. Genet. 48, 848-855 (2016).

27. Bahreini, A. et al. Mutation site and context dependent effects of ESR1 mutation in genome-edited breast cancer cell models. Breast Cancer Res. 19, 60 (2017).

28. Fanning, SW, et al. Estrogen receptor alpha somatic mutations Y537S and D538G confer breast cancer endocrine resistance by stabilizing the activating function-2 binding conformation. Elife 5, https://doi.org/10.7554/eLife.12792 (2016).

29. Harrod, A. et al. Genomic modelling of the ESR1 Y537S mutation for evaluating function and new therapeutic approaches for metastatic breast cancer. Oncogene 36, 2286-2296 (2017)
30. Bose, R. et al. Activating HER2 mutations in HER2 gene amplification negative breast cancer. Cancer Discov. 3, 224-237 (2013).

31. Ben-Baruch, N. E., Bose, R., Kavuri, S. M., Ma, C. X. \& Ellis, M. J. HER2-mutated breast cancer responds to treatment with single-agent neratinib, a secondgeneration HER2/EGFR tyrosine kinase inhibitor. J. Natl. Compr. Canc. Netw. 13, 1061-1064 (2015).

32. Gonzalez-Perez, A. et al. IntOGen-mutations identifies cancer drivers across tumor types. Nat. Methods 10, 1081-1082 (2013).

33. Jeanes, A., Gottardi, C. J. \& Yap, A. S. Cadherins and cancer: how does cadherin dysfunction promote tumor progression? Oncogene 27, 6920-6929 (2008).

34. Stafford, L. J., Vaidya, K. S. \& Welch, D. R. Metastasis suppressors genes in cancer Int. J. Biochem. Cell Biol. 40, 874-891 (2008).

\section{c) (i)}

Open Access This article is licensed under a Creative Commons Attribution 4.0 International License, which permits use, sharing, adaptation, distribution and reproduction in any medium or format, as long as you give appropriate credit to the original author(s) and the source, provide a link to the Creative Commons license, and indicate if changes were made. The images or other third party material in this article are included in the article's Creative Commons license, unless indicated otherwise in a credit line to the material. If material is not included in the article's Creative Commons license and your intended use is not permitted by statutory regulation or exceeds the permitted use, you will need to obtain permission directly from the copyright holder. To view a copy of this license, visit http://creativecommons. org/licenses/by/4.0/.

(c) The Author(s) 2018 